Revue d'histoire de l'enfance « irrégulière »

Le Temps de l'histoire

7 | 2005

Enfermements et éducations

\title{
A la marge des prisons pour mineurs : les prisons- écoles, des structures carcérales à vocation éducative et professionnalisante
}

Élise Yvorel

\section{(2) OpenEdition \\ Journals}

Édition électronique

URL : http://journals.openedition.org/rhei/1059

DOI : 10.4000/rhei.1059

ISBN : 978-2-7535-1645-8

ISSN : $1777-540 \mathrm{X}$

Éditeur

Presses universitaires de Rennes

Édition imprimée

Date de publication : 15 novembre 2005

Pagination : 17-40

ISSN : 1287-2431

Référence électronique

Élise Yvorel, «A la marge des prisons pour mineurs : les prisons-écoles, des structures carcérales à vocation éducative et professionnalisante », Revue d'histoire de l'enfance «irrégulière » [En ligne], 7| 2005, mis en ligne le 06 juin 2007, consulté le 04 décembre 2020. URL : http:// journals.openedition.org/rhei/1059; DOI : https://doi.org/10.4000/rhei.1059 


\section{À la marge des prisons pour mineurs : $\quad$ Élise Yvorel ${ }^{(1)}$ les prisons-écoles, des structures carcérales à vocation éducative et professionnalisante}

\section{La grande diversité des prisons pour mineurs}

Dans la perspective chronologique de l'ensemble du XXème siècle, le terme de " prison " englobe des réalités complexes à cerner. La définition du Larousse n'est pas suffisante, même si, par essence, elle figure l'acception générale de ce mot. Il est indéniable que les prisons sont, en large majorité, des «établissements pénitentiaires où sont détenus les personnes condamnées à une peine privative de liberté ou en instance de jugement $"{ }^{(2)}$ Nous pouvons parler, afin de désigner ce type d'établissement, de " prison ordinaire ", expression empruntée à un juriste du XIXème siècle. ${ }^{(3)}$ L'imaginaire renvoie alors, à juste titre, à de hauts murs qui encerclent des bâtiments massifs, gris, aux fenêtres munies de barreaux.

La prison c'est effectivement cela mais, quand il s'agit de la prison pour mineurs, d'autres réalités sont à prendre en considération. Cette représentation est celle des maisons d'arrêt et des maisons centrales qui, pour la première tout du moins, ${ }^{(4)}$ voient effectivement passer, depuis plus d'un siècle, la majorité des jeunes incarcérés, condamnés ou prévenus. Pourtant, en ce qui concerne les moins de 18 ans, la situation carcérale recouvre de multiples réalités bien plus difficilement décelables. Toutes les institutions qui accueillent les mineurs de justice, si elles peuvent être regroupées sous l'appellation "prisons », ne sont pas forcément reconnues comme telles et n'en possèdent souvent pas officiellement le statut, fenêtres grillagées ou pas.

En effet, la conscience d'avoir à apporter un traitement pénal particulier à cette catégorie juridique a engendré moult expériences. Des essais sont entrepris vers des établissements spécialisés à caractère plus ou moins répressif, sous l'administration directe tantôt de la " pénitentiaire ", tantôt de l'Éducation surveillée, dans des locaux hermétiques ou offrant une certaine ouverture,
(1) Docteur en histoire, université de Poitiers.

(2) Petit Larousse en couleur, Larousse, 1991, p. 800 .

(3) Jacques Bonzon, La législation de l'enfance, Paris, librairie Guillaumin et Cie, 1894, p. 110.

(4) Les maisons centrales n'accueillent les jeunes délinquants, à de rares exceptions, qu'une fois leur majorité atteinte.

Élise Yvorel / p. 17 à 40 
(5) Ludivine Bantigny, Le plus bel âge? Jeunes, institutions et pouvoirs en France des années 1950 au début des années I960, thèse d'histoire, IEP de Paris, 2003, 913 p. (notamment le chapitre premier).

(6) Cette préoccupation n'est cependant pas entièrement nouvelle. Une note ministérielle en date du 26 septembre 1860 préconisait déjà d'établir des quartiers spéciaux pour les jeunes adultes. Instructions du 26 septembre 1860 relatives à l'établissement de quartiers spéciaux pour les jeunes adultes dans les prisons affectées aux hommes, Code pénitentiaire, tome III, p. 146. avec des encadrements éducatifs et/ou disciplinaires. Sont nés ainsi, depuis le milieu du XIXème siècle, des lieux d'enfermement hybrides, à mi-chemin entre volonté d'adoucissement de la peine et redressement sévère de l'individu. Réservés aux enfants, ils ont une finalité souvent floue et, la plupart du temps, jamais atteinte.

En résumé, au XIXème et au XXème siècles, on dénombre, en France, trois modes de prise en charge des mineurs de justice :

- la prison " ordinaire " (maisons centrales, maisons d'arrêt...) ;

- les établissements spécifiques, parfois très proches de la prison, mais qui different de cette dernière par une histoire particulière (colonies agricoles et industrielles, institutions religieuses, institutions publiques d'éducation surveillée...) ;

- le milieu ouvert (patronages, liberté surveillée...).

Après 1945, sans remettre la prison en cause, l'Administration pénitentiaire centre davantage son action vers l'amendement et l'éducatif. Paul Amor est chargé de mettre en place ces nouvelles orientations : il propose alors une réforme en quatorze points.

\section{L'émergence d'une nouvelle catégorie de détenus : les jeunes majeurs}

$\mathrm{Au}$ lendemain de la seconde guerre mondiale, la sauvegarde et la protection de la jeunesse sont l'une des priorités de l'État. L'ordonnance de 1945 et la création de l'Éducation surveillée semblent régler le sort des plus jeunes, les mineurs pénaux de 18 ans, et vouloir les faire sortir des prisons. Ces derniers sont censés bénéficier de mesures éducatives et non plus de répression par le tout carcéral. Leur incarcération doit être exceptionnelle et dûment justifiée. Désormais, ils ne doivent être confiés qu'exceptionnellement à l'Administration pénitentiaire qui, jusque-là, prenait en charge, directement ou dans les établissements privés qu'elle contrôlait, la presque totalité des mineurs de justice.

L'après-guerre est marqué par l'émergence de la notion de jeunesse et toute une réflexion sur la post-adolescence voit le jour. ${ }^{(5)}$ Ainsi, si la loi fixe la majorité à 18 ans, les législateurs sont d'accord pour penser qu'un jeune homme ou une jeune fille reste particulièrement influençable, en bien comme en mal, jusqu'à ses 22 ans. Le sort pénal à réserver à cette nouvelle 
catégorie de détenus est donc au cour des préoccupations. ${ }^{(6)}$ L'Administration pénitentiaire désire créer des structures spécifiques, quitte à oublier pour un moment les mineurs pénaux, qui, théoriquement, doivent être à la charge exclusive de l'Éducation surveillée. Ainsi, en 1953, un rapport ${ }^{(7)}$ du juge Chazal et du criminologue Pinatel propose une base à l'élaboration d'un statut de jeune adulte délinquant. Lâge pour le définir irait de 18 à 25 ans et certains des principes avancés par ces deux rédacteurs sont bien inspirés de la législation qui s'applique déjà aux moins de 18 ans. Quelques-unes des suggestions sont représentatives : le jeune majeur pénal « échappe à toutes sanctions répressives"; sa peine doit être réduite par rapport à celle encourue par les adultes; il bénéficie de la disposition selon laquelle « sont interdites toutes publications de compte rendus des débats et de tous articles concernant les jeunes adultes poursuivis »; un tribunal spécial lui est réservé ; il «ne doit être placé provisoirement en maison d'arrêt qu'en cas de nécessité »... En attendant l'adoption d'un statut juridique particulier pour ces " jeunes majeurs ", les législateurs vont d'ores et déjà régler le problème de leur placement pénal.

Pour ce faire, l'Administration pénitentiaire va associer deux principes fondamentaux. L'un est tout droit sorti de la réforme Amor : " La peine privative de liberté a pour but essentiel l'amendement et le reclassement social du condamné ». ${ }^{(8)}$ Le second provient davantage des nouvelles conceptions de la jeunesse : le jeune majeur condamné, de par son âge, est susceptible de recevoir avec profit une éducation professionnelle, physique et morale adaptée à sa condition de jeune adulte et différente des pratiques exercées sur les adultes. La combinaison de ces deux idées amène à la conclusion qu'il faut rapidement prendre des dispositions pour cette catégorie spécifique, à qui l'on doit donner les moyens de se réinsérer socialement au sortir de la peine de privation de liberté. Puisque la législation sur les tribunaux pour enfants n'est pas applicable au jeune majeur, qu'il ne peut être envoyé dans un établissement d'éducation surveillée et qu'il est en principe soumis au même régime que les adultes, Paul Amor et ses collaborateurs vont donc inventer un nouveau type d'institution spécialement pour cette catégorie de contrevenants à la loi : les prisons-écoles de l'Administration pénitentiaire. Fortement inspirées du modèle de la prison-école belge qui fonctionne depuis 1923, ${ }^{(9)}$ ces institutions françaises sont d'abord ouvertes, puis fermées.
(7) Jean Chazal, Jean

Pinatel, « Séance de section du 19 décembre 1953 : Rapport sur le statut des jeunes adultes délinquants ", Revue pénitentiaire et de droit pénal, $\mathrm{n}^{\circ} 1$ à 3 , janviermars 1954, p. 33-44.

(8) Principe formulé en mai 1945 par la commission de réforme des institutions pénitentiaires françaises.

(9) A. M. Delerneux, « La prison-école par A. M. Delerneux, directeur adjoint de l'établissement pénitentiaire à Merxplas. Extrait de la Revue de droit pénal et de criminologie et Archives internationales de médecine légale ", Vve Ferdinand Larcier, Bruxelles, 1923, $34 \mathrm{p}$. 
(10) Extrait du Rapport d'ensemble présenté par l'inspection générale de l'administration. 1949-1950, ministère de l'Intérieur, Paris, 1951, p. 55.

(11) Oermingen fonctionnait en fait déjà comme centre pénitentiaire pour des condamnés adultes de cours de justice, depuis novembre 1946. S'il ne devient autonome qu'en janvier 1948 , il avait reçu, dès septembre 1947, un contingent de jeunes gens.

(12) «Conseil supérieur de l'Administration pénitentiaire - Séance du 30 janvier 1947. Rapport de M. Amor, directeur de l'AP - Présidence de M. André Marie, garde des Sceaux, ministre de la Justice " + Annexes, Revue pénitentiaire et de droit pénal, n 4-5-6, avrilmai-juin 1947, p. 184.

(13) Extrait du Rapport d'ensemble présenté
Après 1945, que ce soit pour les mineurs pénaux ou pour les jeunes majeurs, l'Administration pénitentiaire s'évertue à mettre en place des systèmes qui permettent à cette " jeunesse dévoyée " d'acquérir quelques bases scolaires et professionnelles, afin que la réinsertion soit facilitée au terme de la peine. Dans l'esprit des législateurs, ce but ne peut être atteint que par l'élaboration d'un emploi du temps "plein " : il s'agit d'occuper en permanence, par des activités éducatives diverses, les jeunes corps et les jeunes esprits. Les prisons-écoles pour garçons d'Oermingen et de Loos-lez-Lille entrent parfaitement dans cette double problématique interactive et sont d'ailleurs élaborées sur ce modèle. Cependant, elles vont rapidement échouer dans leur premier principe : conçues, à l'origine, exclusivement pour les 18-28 ans, elles accueillent pourtant, selon un processus maintes fois répété dans l'histoire de la jeunesse en prison, des adolescents qui n'ont pas encore atteint leur majorité. Dans leur second principe, qui mise sur une formation scolaire et professionnelle et sur des journées remplies, elles ne vont pas être non plus une totale réussite, ni pour les adolescents mineurs, ni pour les jeunes majeurs.

\section{Quand les mineurs font leur entrée chez les jeunes majeurs... faute de mieux}

1.1. De la création des prisons-écoles d'Oermingen et de Loos-lez-Lille

La première prison-école pour garçons de l'histoire de France, « le premier établissement fondé dans l'esprit de la réforme " ${ }^{(10)}$ ouvre ses portes à la fin de l'année 1947, à Oermingen dans la Moselle, dans une ancienne caserne cédée par le ministère de la Guerre. ${ }^{(11)}$ Il est prévu que, pour cet établissement, « il sera fait appel essentiellement à la formation professionnelle par l'apprentissage, [...] mais aussi et surtout la rééducation et le reclassement social ", ${ }^{(12)} \mathrm{Et}$, en effet, lors de son autonomie définitive le 1er janvier 1948, "Oermingen, dans l'ensemble de la réforme pénitentiaire, est réservé aux jeunes condamnés de 18 à 25 ans, primaires ou récidivistes, n'ayant pas à subir en principe de peine supérieure à 5 ans de réclusion. Ces jeunes détenus sont soumis à quatre phases successives de rééducation avant d'atteindre leur mise en liberté définitive, soit sous forme de libération conditionnelle, soit en fin de peine. " ${ }^{(13)}$ C'est donc sous le signe d'un régime sélectif et progressif que sont censés évoluer ces jeunes gens. Ce schéma de fonctionnement type, repris par Paul Amor pour l'élabo- 
ration du système progressif dans les centrales, ${ }^{(14)}$ est fortement empreint du règlement des maisons d'éducation surveillée. Le rapport d'inspection de l'Administration pénitentiaire pour les années 1949 et 1950, ainsi qu'un article du directeur de la dite prison-école, A. Gayraud, de 1952, ${ }^{(15)}$ nous permettent d'en résumer le fonctionnement selon ses quatre phases distinctes.

La première phase, celle d'observation, consiste en un isolement de trois mois à Rethel, ${ }^{(16)}$ " centre d'attente et de triage où les jeunes condamnés, soumis à la plus austère discipline pénitentiaire, vivent isolément dans des cellules tristes, voûtées, aux petites lucarnes haut placées et armées de barreaux ». (17) S’ils " ne se révèlent pas indignes ou non susceptibles de bénéficier de l'école ", ${ }^{(18)}$ l'observation se poursuit à Oermingen dans un pavillon isolé où " les fenêtres sont sans barreaux. Au-delà d'une faible barrière en barbelés, la vue s'étend sur la campagne. Déjà on oublie la prison ». ${ }^{(19)}$ Pendant cette période, le jeune majeur est toutefois mêlé aux autres pensionnaires par des cours en commun d'éducation physique et d'instruction générale, mais il n'est astreint à aucun travail : "Il est soumis à l'observation quotidienne d'un éducateur et à l'observation intermittente du directeur, du sous-directeur, de l'assistante sociale, du juge présidant la commission de classement, du surveillant-chef, du médecin et des divers ministres du culte " ${ }^{(20)}$ afin, entre autres, que puissent être détectées ses aptitudes particulières.

La seconde phase est celle de l'éducation, autrement dit, celle de l'apprentissage professionnel qui débouche sur l'obtention d'un CEP ou d'un CAP. Ce travail s'effectue en commun par groupe de trente environ, sauf la nuit, pendant laquelle les jeunes sont répartis dans des dortoirs cellulaires, sous la surveillance des éducateurs qui les ont suivis pendant la première période d'observation. Les jeunes gens peuvent alors choisir entre plusieurs ateliers suivant leurs aptitudes. À l'ouverture de la prison-école, sept ateliers sont déjà en état de fonctionnement : menuiserie, mécanique générale, ajustage, métaux en feuilles, forge, maçonnerie et cordonnerie. ${ }^{(21)}$ Somme toute, nous retrouvons là les activités offertes aux plus jeunes dans les maisons d'éducation surveillée. La classe, quant à elle, est assurée le plus souvent par des détenus adultes, sachant que les textes de loi ne prévoient pas, contrairement à ceux qui s'appliquent aux mineurs, de séparation entre jeunes adultes et autres détenus. Cette phase dure un minimum de six mois. Ce délai expiré, une par l'inspection générale de l'administration. 1949-1950, ministère de l'Intérieur, Paris, 1951, p. 55.

(14) Direction de l'Administration pénitentiaire, « La réforme Amor et sa mise en œuvre dans l'immédiat après-guerre, (1944-1950) ", La lettre des cadres, $\mathrm{n}^{\circ} 29$, semaine $\mathrm{du}$ 17 au 21 juin 2002, 2 p.

(15) A. Gayraud, «La prison-école d'Oermingen ", Revue pénitentiaire et de droit pénal, $\mathrm{n}^{\circ} 10$ à 12 , octobre-décembre 1952, p. 680-689.

(16) Cette " annexe " à la prison-école est supprimé en 1956, suite à l'ouverture d'une section d'apprentissage de peinture dans les locaux d'Oermingen, qui a permis une augmentation de l'effectif.

Robert Lhez, Rapport général présenté à monsieur le garde des Sceaux par 
Robert Lhetz, directeur de

l'Administration

pénitentiaire, ministère de

la Justice, Paris, exercice

1956, p. 61.

(17) A. Gayraud, op.

cit., p. 680.

(18) Ibid., p. 680.

(19) Extrait du Rapport d'ensemble présenté par l'inspection générale de l'administration. I949-I950, ministère de l'Intérieur, Paris, 1951, p. 55.

(20) Ibid., p. 55.

(21) En 1974, à

l'époque où est abandonnée l'appellation «prisonécole ", des ateliers de plâtrerie, limousinerie, béton armé, plomberie, chauffage central, peinture, électricité, menuiserie, soudage, tournage, fraisage et jardinage se sont substitués à ces sept ateliers.
(22) Extrait du Rapport d'ensemble présenté par l'inspection générale de l'administration. 1949-I950, ministère de l'Intérieur, Paris, 1951, p. 55.

(23) Ibid., p. 57.

(24) Ibid., p. 57.

(25) Ibid., p. 57.

(26) Ibid., p. 59.

(27) Pierre Orvain,

Direction de

l'Administration péniten-

tiaire, exercice 1960.

Rapport général présenté à

monsieur le garde des

Sceaux par Pierre Orvain,

directeur de l'Adminis-

tration pénitentiaire,

ministère de la Justice,

Paris, 1961, p. 25.

(28) Archives contem-

poraines de

Fontainebleau, 960136,

individualisation du traitement, art. 66, procédure de classification, « Note de service du directeur du Bureau de l'application des peines, R. Lut, aux directeurs régionaux à commission de classement se réunit afin de déterminer si « les signes d'amendement que manifeste le jeune détenu lui permettent d'entrer dans la troisième phase que l'on nomme la phase de confiance $" .^{(22)}$

Cette avant-dernière étape n'est guère différente de la précédente. Seuls quelques avantages matériels sont concédés, tels que la chambre individuelle dotée d'un lavabo, l'uniforme neuf, le parloir libre et l'accès, le samedi, à la TSF. De plus, les détenus peuvent obtenir des suppléments alimentaires " par leur propre travail, dans un jardin mis à leur disposition et par un clapier qu'ils peuvent entretenir ». ${ }^{(23)}$ Lors de la phase de confiance, les plus méritants, "qui se trouvent à trois mois de leur libération définitive ou à trois mois de leur libération conditionnelle à terme préfixé » et dont « la récidive est peu probable et l'apprentissage professionnel terminé ", ${ }^{(24)}$ peuvent éventuellement intégrer la dernière phase, celle de semi-liberté, dont le home est à Nancy. Ils sont alors placés en atelier et à l'usine et ne restent au home que les soirées et le week-end.

La conclusion de cette description du fonctionnement interne de la prison-école est " peut-on en faire plus ? ". ${ }^{(25)}$ Mais la suite de ce rapport, plus analytique, précise qu'il est " nécessaire de perfectionner la sélection à l'entrée pour ne recevoir que des éléments vraiment susceptibles de bénéficier du régime de l'école » et qu'il conviendra à l'avenir « d'exclure les jeunes qui se révèlent à l'observation vraiment inéducables ».(2) C'est, entre autres, cette requête qui va amener les directeurs de l'Administration pénitentiaire à ouvrir de nouvelles prisons-écoles légèrement différentes. En effet, Oermingen ne donne pas tous les résultats escomptés.

Le rapport général de fonctionnement de 1960 nous apprend que l'Administration pénitentiaire a effective- 
ment pris en considération le fait qu'il est nécessaire de s'occuper des jeunes adultes qui ne peuvent être affectés à la prison-école d'Oermingen pour diverses raisons :

"Il s'agit parfois de jeunes délinquants qui ne possèdent pas les connaissances scolaires de base nécessaires pour suivre avec profit un enseignement scolaire, ou encore de condamnés qui devront être élargis antérieurement à l'examen qui sanctionnera leurs études.

Mais ce sont, le plus souvent, des éléments assez difficiles, que leur comportement rend indésirables dans un établissement ouvert. " ${ }^{(27)}$

Qu'à cela ne tienne, l'Administration pénitentiaire va créer des prisons-écoles fermées. La première est aménagée à la maison centrale de Toul d'après une décision de janvier $1958,{ }^{(28)}$ mais elle ne survit que très peu de temps, car l'établissement est réquisitionné, en décembre 1959, pour les détenus nordafricains, nombreux en cette période de guerre d'Algérie. ${ }^{(29)}$ Il est donc aménagé, à la place, un centre de jeunes condamnés dans les centrales de Melun et d'Ensisheim. Cependant, ces centres ne font pas bénéficier leurs pensionnaires du régime progressif, mais seulement d'un « traitement rééducatif, auquel participent les éducateurs ». ${ }^{(30)}$

Le reste du temps, ils travaillent dans les mêmes ateliers que les adultes de la centrale, et c'est pour cela que ces institutions, assez éphémères, ne recevront jamais de mineurs de 18 ans, contrairement aux prisons-écoles. Au sujet des majeurs pénaux âgés de moins de 21 ans, " toutes facilités doivent leur être données pour qu'ils puissent profiter d'un enseignement scolaire et professionnel ", ${ }^{(31)}$ même s'ils sont jugés trop difficiles pour un régime basé sur l'ouverture. Comme ce précepte législatif paraît bien difficile à appliquer dans les maisons d'arrêt surpeuplées, qu'Oermingen tend de plus en plus vers l'ouverture et que la centrale de Toul n'est plus disponible, l'administration va ouvrir la prison-école fermée de Loos-lez-Lille en 1961. Prison centrale cellulaire déjà pourvue de vastes ateliers, elle semble parfaitement convenir pour l'usage que l'Administration pénitentiaire désire en faire. De trois cent soixante places initiales, elle passe à quatre cent dix à compter de l'exercice 1966. Cette institution possède à peu près le même fonctionnement interne, progressif, que sa grande sœur ouverte, même si les ateliers de formation sont bien moins nombreux ${ }^{(32)}$ et la surveillance plus drastique : propos de la classification des jeunes condamnés ", 9 janvier 1958.

(29) Finalement, libéré de ses activistes au début de l'année 1966, Toul aura un quartier cellulaire affecté à l'incarcération des jeunes détenus en provenance de Paris, mais uniquement ceux âgés de 21 à 23 ans au moment de l'affectation ou ayant un reliquat de peine de 6 mois à 1 an à subir. Le 15 septembre de la même année est ouvert le centre de jeunes détenus condamnés à de courtes peines (moins de 6 mois) d'Ecrouves, où sont affectés essentiellement de jeunes parisiens. Archives contemporaines de Fontainebleau, 960136, individualisation du traitement, art. 66, procédure de classification, « Note sur l'amélioration des conditions de détention des jeunes détenus ", n.d. (estimation date : 1967). 
(30) Pierre Orvain, op. cit., p. 25.

(31) Art. D.450 du Code de procédure pénale.

(32) En 1974, juste avant l'abandon du terme de "prison-école ", Loos ne compte que trois ateliers : électricité de bâtiment, serrurerie de bâtiment et mécanique automobile.

(33) Archives contemporaines de Fontainebleau, 960136, individualisation du traitement, art. 66, procédure de classification, "Note sur l'amélioration des conditions de détention des jeunes détenus ", n.d. (estimation date : 1967).
(34) Archives contemporaines de Fontainebleau, 960136, catégories pénitentiaires, art. 45, mineurs, "Étude sur le problème posé par les jeunes détenus incarcérés après échec de leur traitement dans les institutions pour mineurs délinquants. Situation à la prison-école d'Oermingen, lettre du directeur de l'institution au garde des Sceaux ", 28 avril 1969.

\section{(35) Désormais deux} distinctions sont faites parmi les jeunes de plus de 18 ans : les « majeurs pénaux " ont entre 18 et 21 ans et les " jeunes adultes » ont entre 21 et 23 ans.

(36) Archives contemporaines de Fontainebleau, 960136, individualisation du traitement, art. 66, procédure de classification, "Le garde des Sceaux aux procureurs généraux. Objet : Prisons-
"La maison centrale de Loos qui dispose d'un personnel spécialisé fonctionne selon le régime de la prison-école et reçoit des condamnés à des peines assez longues (trois ans et plus). Les jeunes sont soumis à un régime éducatif axé selon leur personnalité sur le travail pénal, l'enseignement scolaire ou la formation professionnelle. Ils bénéficient d'activités sportives et récréatives. " (33)

En 1969, le directeur d'Oermingen ne s'y trompe d'ailleurs pas lorsqu'il préconise, à l'occasion d'une enquête sur les anciens de l'Éducation surveillée, réputés détenus très difficiles :

"Pour ma part, j'estime que ce type de délinquant n'a pas sa place dans l'établissement, dont le régime s'apparente à celui d'une IPES, sans pour autant les condamner à la prison classique.

Il semble qu'un système plus viril soit à conseiller, la prison-école fermée par exemple. " ${ }^{(34)}$

Si nous avons fait cette incursion dans le monde carcéral des jeunes adultes, aménagé par l'Administration pénitentiaire, si nous nous sommes attachée à montrer la genèse et la base du fonctionnement d'Oermingen et de Loos, c'est que, l'une et l'autre, la « douce " et la "sévère ", pourtant toutes deux conçues pour les majeurs pénaux, ${ }^{(35)}$ vont accueillir des mineurs pénaux.

\subsection{Des adolescents mineurs dans les prisons- écoles pour jeunes majeurs}

Dès le projet de réforme Amor, il est évident que les prisons-écoles sont conçues pour les jeunes adultes, ceux qui ne peuvent être jugés par les juridictions pour enfants. La circulaire du 16 septembre 1951 portant création d'Oermingen et de Doullens, on ne peut plus claire sur ce point, est rappelée quelques jours plus tard 
aux procureurs généraux par le garde des Sceaux :

«[...] je vous ai informé de la création de deux prisons-écoles à Oermingen et à Doullens affectées respectivement à la détention des jeunes condamnés de l'un et l'autre sexe, majeurs selon la loi pénale. " (36)

Le directeur d'Oermingen, en 1952, a d'ailleurs totalement intégré cette donnée : "L'âge des intéressés est toujours situé entre 18 et 25 ans ; c'est de règle. La prison-école est destinée à recevoir les condamnés majeurs selon la loi pénale et dont la peine doit venir à expiration avant qu'ils n'aient atteint l'âge de 25 ans. En deçà de ces limites, il s'agirait d'un mineur relevant des services de l'Éducation surveillée, au-delà, d'un adulte dont la destination pénale est une maison centrale. " ${ }^{(37)}$

Or, s'ils ne sont jamais majoritaires dans ces institutions, les moins de 18 ans sont toutefois rapidement bel et bien présents. Notons, d'ores et déjà, que nous ne retrouvons pas à Oermingen et à Loos de jeunes prévenus, mais uniquement des jeunes condamnés ; en cela au moins, le principe qui consiste à réserver ces institutions exclusivement à des jeunes déjà jugés est-il respecté. Le processus qui conduit des enfants n'ayant pas encore acquis leur responsabilité pénale dans les prisons-écoles relève d'un choix délibéré de l'Administration pénitentiaire. En effet, un jeune condamné à un enfermement de plus de douze mois dans le cadre des dispositions de l'ordonnance du 2 février 1945 doit normalement exécuter sa peine d'emprisonnement dans une institution spéciale relevant de l'Éducation surveillée. ${ }^{(38)}$ Si le reliquat de peine est inférieur à douze mois, le mineur pénal est affecté à un quartier spécial de maison d'arrêt. Or, les institutions spéciales pour les condamnés de moins de 18 ans n’ont jamais été créées et les quartiers pour mineurs, lorsqu'ils existent, sont surpeuplés, déjà occupés par les petits prévenus et courtes peines. Une note distribuée par l'Administration pénitentiaire en 1963 explique la politique qu'elle adopte pour les mineurs de 21 ans, mineurs pénaux compris :

"Les institutions spéciales de l'Éducation surveillée n’ont pas été créées. Les mineurs, au sens de l'ordonnance du 2 février 1945, suivent en conséquence le sort des majeurs pénaux de 18 à 21 ans et des jeunes adultes [...] L'Administration pénitentiaire considérant qu'il convient, indépendamment de leur situation juridique [...] de soumettre tous les jeunes de 21 ans à un régime approprié, a pu avec les moyens réduits dont elle dispose actuelle-

écoles ", n.d. (estimation date : fin octobre 1951).

(37) A. Gayraud, $o p$. cit., p. 682.

(38) Décret du 12 avril 1952 relatif à l'exécution des peines d'emprisonnement concernant les mineurs et créant les Institutions spéciales d'éducation surveillée pour les mineurs les plus difficiles, Bulletin de l'Union des sociétés de patronage de France, $\mathrm{n}^{\circ} 14$, 2ème trimestre 1952, p. 391-393. 
(39) Ce quartier, dit des

" J.3 ", est à ne pas confondre avec le Centre spécial d'observation de l'Éducation surveillée de Fresnes (CSOES). Ce n'est pas un centre d'observation, mais bien un quartier pour prévenus et condamnés ; le personnel dépend de l'Administration pénitentiaire et il reçoit essentiellement des majeurs pénaux même si l'on peut noter la présence de quelques mineurs. Ainsi, en 1966, dix-sept garçons de moins de 18 ans sont incarcérés auprès des cinq cent vingt-deux majeurs pénaux.

(40) Archives contemporaines de

Fontainebleau, 960136, catégories pénitentiaires, art. 45, mineurs, "Note sur la détention des mineurs de 21 ans dans les établissements pénitentiaires ", note distribuée par l'Administration pénitentiaire, 6 décembre 1963, p. 5.

(41) Ibid., p. 8.

(42) Souligné dans le texte d'origine.

(43) Les passages soulignés, le sont dans la lettre manuscrite originale.

Archives contemporaines de Fontainebleau, 960136, individualisation du traitement, art. 66 , procédure de classification, «Lettre du conseiller de la cour d'appel d'Aix délégué à la protection de l'enfance et aux œuvres post-pénales au garde des Sceaux », 10 octobre 1960.

ment en locaux et en personnel ouvrir des quartiers de mineurs, notamment à Fresnes. ${ }^{(39)}$

Ces quartiers renferment des prévenus et des condamnés à une courte peine. Les condamnés à une longue peine sont en effet dirigés suivant leur personnalité, soit sur la prison-école ouverte d'Oermingen, soit sur un établissement à régime progressif, soit encore sur la prison-école fermée de Loos (dans la mesure des places disponibles). " ${ }^{(40)}$

C'est donc parce qu'il entre « dans la mission normale de l'Administration pénitentiaire d'assurer l'exécution des peines d'emprisonnement, à charge pour elle, comme elle le fait d'ailleurs en fait, d'adapter le régime des jeunes condamnés de manière à leur fournir les moyens éducatifs et les activités convenant à leur âge ", ${ }^{(41)}$ qu'elle choisit d'envoyer des condamnés de moins de 18 ans à de longues peines en prisons-écoles. Leur placement dans un établissement à régime progressif ou dans les centres de Melun et d'Ensisheim est en effet exclu, puisqu'ils y seraient en compagnie de détenus adultes, situation législativement maintes fois réprouvée. Cette situation n'est cependant pas sans créer quelques confusions qui perdurent plus de dix ans après l'ouverture de la première prison-école. Ainsi, malgré un texte clair, des tribunaux pour enfants, des juges d'instruction ou des conseillers de cour d'appel, s'y perdent. Pour preuve, cette lettre adressée au garde des Sceaux par l'un de ces derniers, en octobre 1960 :

" [...] la Chambre des mineurs de la cour est saisie d'un appel concernant le jeune C.G.

Ce garçon est né le 8 avril 1943 à Saint-Jean-d'Angély, donc âgé actuellement de 17 ans, a été condamné par le TE de Nice le 16 juin et le 11 août 1960 à deux années d'emprisonnement ${ }^{(42)}$ pour vols, tentatives de vols, port d'arme, etc. 
La cour qui doit rendre son arrêt le 7 novembre prochain aimerait connaître quel serait le régime pénitentiaire auquel serait soumis ce garçon, si la peine était confirmée ou même portée à trois ans d'emprisonnement. Elle aimerait savoir en particulier si C.G. pourrait bénéficier de l'enseignement d'une prison-école. " ${ }^{(43)}$

Un second exemple émane cette fois d'un juge d'instruction, via une lettre adressée à la direction de l'Éducation surveillée en juillet 1961 :

« [...] informe contre le nommé G.A., mineur de 18 ans comme étant né le 19 mai 1944, poursuivi pour une agression commise en compagnie d'un sieur D. sur un passant dans l'intention de le voler.

J'envisage de le renvoyer devant la chambre d'accusation de la cour d'appel de Douai pour être traduit devant la cour d'assises des mineurs.

J'ai l'honneur de vous prier respectueusement de bien vouloir me faire connaître quel établissement serait susceptible d'accueillir G.A., s'il était condamné par la cour d'assises des mineurs à une peine d'emprisonnement $[\ldots] »{ }^{(44)}$

Adressé à la direction de l'Éducation surveillée, au directeur du bureau de l'application des peines ou directement au garde des Sceaux, ce type de demande de renseignements est fréquent. Il ressort des diverses réponses que, mis à part des conditions de fonctionnement internes spécifiques aux prisons-écoles, rien ne s'oppose effectivement à l'intégration de mineurs pénaux condamnés à de longues peines dans ces établissements. Ainsi, dans la réponse du garde des Sceaux à la première demande, aucune mention n'est faite des 17 ans du jeune C.G., alors que toutes les autres précisions quant à l'admission en prison-école sont données :

" [...] l'admission dans une prison-école suppose la réunion de plusieurs conditions : la durée de la peine restant à subir à partir du moment où la condamnation devient définitive doit être supérieure à un an et le condamné ne devra pas être âgé de plus de vingt-huit ans à la date prévue pour l'expiration de la peine (art. 718 et D. 73 du CPP). De plus, le jeune détenu doit avoir été reconnu capable, du point de vue des autres aspects de sa personnalité, de tirer profit d'un traitement de rééducation et de formation morale, scolaire et professionnelle. " ${ }^{(45)}$

À la même époque, d'autres cependant ne s'y trompent pas. C'est le cas du
(44) Archives contemporaines de Fontainebleau, 96or36, individualisation du traitement, art. 66, procédure de classification, "Lettre d'un juge d'instruction au directeur de l'Éducation surveillée, demande de renseignements sur le cas d'un mineur pénal ", 13 juillet 1961.

(45) Archives contemporaines de Fontainebleau, 960136, individualisation du traitement, art. 66, procédure de classification, "Réponse du garde des Sceaux à M. le Procureur général près de la cour d'appel d'Aixen-Provence ", 21 octobre 1960. 
(46) Souligné dans le texte d'origine.

(47) Archives contemporaines de Fontainebleau, 960136, individualisation du traitement, art. 66, procédure de classification, "Lettre du président du tribunal pour enfants de Marseille au garde des Sceaux.

Objet : Affectation de mineurs en prison école ", 25 juillet 1961.

(48) Archives contemporaines de Fontainebleau, 960136, sûreté et discipline, art. 100, règlement intérieur, "Règlement Mémento à l'usage des détenus - Maison centrale de Loos, centre de jeunes condamnés ", n.d. (estimation date : 1966), p. 1 . président du tribunal pour enfants de Marseille qui adresse une requête au ministre de la Justice pour que soient réservées à sa ville des places en prisonsécoles, les petits délinquants de cette commune étant particulièrement turbulents et... jeunes justement :

" J'ai l'honneur de porter à votre connaissance les faits suivants au sujet d'une question qui ne cesse de me préoccuper :

Le TE des Bouches du Rhône a été amené à prononcer ces derniers mois diverses peines d'emprisonnement de longue durée (entre 20 et 30 mois d'emprisonnement) à l'encontre de jeunes délinquants, en faveur desquels tout avait été tenté sans succès sur le plan éducatif : [suivent les cas de six mineurs effectivement condamnés de 20 à 30 mois].

Le tribunal avait alors le ferme espoir que ces peines seraient exécutées en prison-école.

Les dossiers [...] ont été régulièrement constitués et monsieur le directeur de la maison d'arrêt vient de me faire connaître lors de ma visite du 24 courant que le transferement souhaité n'était réalisable, faute de place.

Je me permets d'attirer tout spécialement et très respectueusement votre attention sur cet état de fait que j'estime préjudiciable à l'intérêt de ces garçons - et qui va à l'encontre du but poursuivi par le tribunal.

Se plaçant sur un plan plus général, votre administration ne pourrait-elle pas réserver ${ }^{(46)}$ à l'avenir et par priorité à notre grande ville de Marseille tenant compte des éléments encore jeunes, troubles et remuants dont elle est composée et qui existent à l'état quasi permanent - un certain nombre de places dans l'une des deux prisons-écoles de notre pays ? "(47)

S'il n'est pas donné satisfaction à cette demande, rien cependant n'empêche ce tribunal d'espérer qu'à l'avenir des places se libèreront pour les mineurs pénaux qu'il condamne à des peines de prison ferme. C'est ainsi, par exemple, qu'au 1er juillet 1966, trente-six mineurs pénaux de 18 ans côtoient soixante et onze mineurs civils de 21 ans dans la prison-école fermée de Loos. La même année, le règlement du centre précise même quelques particularités spécifiques aux mineurs pénaux et reconnaît, de fait, la place qui est faite à cette population particulière. ${ }^{(48)}$ Cependant, il est possible, après tout, que ces jeunes adolescents reçoivent dans les prisons-écoles l'enseignement et l'instruction que les textes législatifs prévoient de leur accorder en cas d'incarcération et qui sont fort peu 
opérationnels dans les autres institutions pour mineurs de justice gérées par l'Administration pénitentiaire, telles les maisons d'arrêt.

2. Les prisons-écoles, institutions de réinsertion socale ? Réussite ou échec? 2.1. Les longues journées professionnalisantes des prisons-écoles : un certain regard sur Loos

La prison-école d'Oermingen est le premier établissement fondé dans l'esprit de la réforme de Paul Amor. C'est donc le premier établissement à mettre en place le tout nouveau régime progressif. Certes, il n'existait pas auparavant d'institution carcérale réservée à cette population et il peut paraître normal qu'une prison pour jeunes détenus ait eu la priorité. Le fait que ce soit une prison-école qui fasse les frais des premiers essais n'est cependant pas innocent. En effet, dans l'esprit des législateurs de l'époque, le jeune homme ou la jeune fille est, plus que les adultes, « influençable en bien comme en mal et susceptible de recevoir avec profit une instruction professionnelle et morale tout particulièrement poussée " ${ }^{(49)}$ Or, dans les quartiers spéciaux pour mineurs des maisons d'arrêt, les activités scolaires et physiques doivent déjà, théoriquement du moins, remplir la majorité du temps quotidien des adolescents, afin qu'ils ne demeurent pas inactifs. Si cette règle n'est ni applicable ni appliquée en ces lieux près des prisons pour adultes, au moins faut-il qu'elle le soit dans ceux conçus tout spécialement pour les plus jeunes, telles les prisons-écoles. D’autant plus que celles-ci sont créées en vue de permettre un " reclassement ", ${ }^{\left({ }^{0}\right)}$ une « réintégration " pour utiliser un terme plus moderne, dans la vie libre. Pour ce, elles appliquent des méthodes de confiance censées laisser une certaine marge de manœuvre aux jeunes et leur faire oublier qu'ils sont en prison. Elles soumettent également les jeunes détenus à " un régime éducatif axé selon leur personnalité sur le travail pénal, l'enseignement scolaire ou la formation professionnelle ", ${ }^{(51)}$ Dans les faits, c'est la formation professionnelle qui a la priorité. Cela est réaffirmé en octobre 1951, à l'ouverture d'Oermingen, par un comité chargé d'une enquête sur l'Administration pénitentiaire et le système répressif :

« [...] en ce qui concerne les détenus eux-mêmes, le Comité estime que leur formation professionnelle constitue l'un des meilleurs éléments de l'œuvre entreprise, leur reclassement définitif dépendant en partie de la
(49) « Conseil supérieur de l'Administration pénitentiaire..., op. cit., p. 184.

(50) A. Gayraud, op. cit., p. 685 .

(51) Archives contemporaines de Fontainebleau, 960136, individualisation du traitement, art. 66, procédure de classification, "Note sur l'amélioration des conditions de détention des jeunes détenus ", n.d. (estimation date : 1967). 
(52) Archives contemporaines de Fontainebleau, 960279, art. 2, dossiers de principes,

"Conclusion sur

l'Administration pénitentiaire et le système répressif ", 26 octobre 1951, p. 18.

(53) Robert Scmelck, Direction de l'Administration pénitentiaire, exercice 1963. Rapport présenté à monsieur le garde des Sceaux, ministre de la Justice par Robert Schmelck, procureur général, directeur de l'Administration pénitentiaire, ministère de la Justice, Paris, 1964.

(54) Ibid., p. 65.

(55) Ibid., p. 66. possibilité où ils se trouvent d'obtenir un emploi à leur libération. Le Comité demande donc que l'apprentissage soit développé. " ${ }^{(52)}$ Or, l'apprentissage d'un métier nécessite de nombreuses heures de travail, théoriques et pratiques. Ce programme est d'autant plus applicable et essentiel dans les prisons-écoles que ces dernières accueillent uniquement des longues peines. Aussi les règlements de la prison-école d'Oermingen et de Loos-lez-Lille prévoient-ils de grandes journées d'activité pour leurs pensionnaires. Nous avons déjà abordé le fonctionnement et le régime de la prisonécole d'Oermingen. Nous allons ici regarder de plus près l'emploi du temps des jeunes gens incarcérés à Loos.

Pour cela, nous nous appuyons essentiellement sur un document qui nous éclaire sur les règles de vie, les horaires et les activités imposés aux jeunes gens. Il s'agit du rapport de fonctionnement annuel de l'Administration pénitentiaire au garde des Sceaux pour l'exercice 1963..$^{(53)}$ Deux ans après l'ouverture du centre, ce rapport constitue « un exposé plus complet sur les particularités du régime de l'établissement et dresse un premier bilan des résultats obtenus ». ${ }^{(54)} \mathrm{Il}$ y a alors trois cent trente-trois pensionnaires dont quatorze mineurs pénaux.

Loos est une prison-école fermée, mais elle est également organisée en vue de l'application d'un régime progressif « classique ». Prévue dans ses statuts et ses fonctions, la mise en place de ce régime inclut la notion d'occupation du temps sans discontinuité. Le rapport de 1963 le confirme clairement :

"Le régime du centre de jeunes condamnés est fondé sur les principes suivants :

- régime cellulaire de nuit ; activités en commun dans la journée ;

- activités des jeunes condamnés orientées vers :

- le travail,

- les cours scolaires,

- les sports,

- les loisirs dirigés ;

- activités soutenues, continues et dirigées tout au long de la journée, afin que les adolescents soient le moins possible livrés à eux-mêmes. " ${ }^{(55)}$ Afin de répondre à ces objectifs, la journée des garçons est basée sur quatorze heures d'activité, soit un lever à 7 h00 et un coucher à 21 h00. Constatons déjà 
que, dans cette fourchette horaire, le retour en cellule pour la nuit est plus tar(56) Ibid., p. 66. dif que dans une prison classique. De plus, 21 h00 n'est pas le moment de l'extinction des feux, mais juste celui de l'entrée en cellule. Plus proche de la vie quotidienne d'un citoyen libre, ce temps en soirée respecte également mieux les besoins biologiques de l'être humain. En effet, l'établissement étant indépendant, il ne relève pas du service d'une maison d'arrêt ordinaire, ce qui permet cette liberté supplémentaire. Cependant, en journée, l'emploi du temps des jeunes gens semble presque chronométré. À chaque action correspond un temps limité, mesuré et compté :

«a) Jours de la semaine (journées scolaires).

- hygiène corporelle, entretien des cellules, repas, repos entre deux activités : 3 heures ;

- travail : 6 à 8 heures, suivant les emplois ;

- cours scolaires : 3 à 6 heures, suivant le niveau des cours ;

- promenades, sports : 1 à 2 heures.

b) Le jeudi et le samedi soir (après 17h00).

- activités à caractère culturel ou distrayant, à la place des cours scolaires.

c) Le dimanche.

- activités sportives, cinéma, théâtre. " ${ }^{(50)}$

Même les loisirs paraissent entrer dans une fourchette de temps prédéfinis. Ainsi, par exemple, si les émissions de radio sont variées (musique moderne, musique classique, théâtre...), le temps d'écoute, lui, est immuable, renouvelé chaque jour, à l'exception du dimanche : il a lieu de $11 \mathrm{~h} 15$ à $12 \mathrm{~h} 15$, et de $21 \mathrm{~h} 00$ à $22 \mathrm{~h} 00$.

Au premier abord, la journée de l'adolescent paraît donc effectivement bien remplie. Toutes les facilités pour occuper son corps et son esprit semblent lui être offertes. D'après Robert Schmeck, l'école pénitentiaire est pleine de promesses et remplit son rôle de formation. Tout va bien, puisque les résultats sont bons. Ainsi, nous pouvons lire au fil des lignes :

"Les résultats aux examens ont été très encourageants »; et

"Les activités sportives ont été développées en priorité et au maximum pour tenir compte des grands besoins de dépenses d'énergie ressentis par ces adolescents détenus. [...] Les résultats obtenus par les pratiquants sont satisfaisants »; 
(57) Ibid., p. 68 et 69.

(58) Ibid., p. 65.

(59) Ibid., p. 67.

(60) Ibid., p. 67.

Les deux cent quatrevingts jeunes correspondent aux condamnés de moins de 25 ans. En effet, les jeunes adultes qui ont atteint cet âge ne bénéficient pas des cours scolaires.

(61) Ibid., p. 68. ou encore

"Toutes ces troupes [d'acteurs bénévoles invités] ont obtenu un succès magnifique et - il faut le souligner - un succès imprévu, car le genre de spectacles offerts ne semblaient pas toujours à la portée de ces adolescents non préparés à des programmes de cette qualité. "(57)

De même, les pensionnaires de l'établissement paraissent contents de leur sort, ou du moins confiants :

"Il est encourageant de constater que les jeunes se montrent sensibles aux efforts qui ont été faits en leur faveur. " ${ }^{(58)}$

Pourtant, sans mettre en doute certains bons résultats d'éléments motivés, une lecture plus attentive du rapport nous fait douter de la réalité de cette activité soutenue. En effet, dans un premier temps, nous apprenons que seuls deux cent vingt-huit garçons ont un travail (dont cinquante-cinq détenus employés aux services généraux). Que font les cent cinq adolescents absents des chantiers et ateliers ? Sont-ils en classe ? Oui pour ceux qui suivent des études supérieures, ainsi que pour les élèves de troisième qui se préparent au BEPC. Mais cela ne peut être le cas pour les autres. En effet, même si l'on exclut les détenus de plus de 25 ans qui n'ont pas accès aux cours, les sept classes de différents niveaux que possède l'école n'ont lieu qu'après la journée de travail ; elles "fonctionnent régulièrement tous les soirs, entre 16 et 21 heures ". ${ }^{(59)}$ La cinquantaine de jeunes de moins de 25 ans qui ne travaillent pas n’a donc pas cours en journée. De plus, " aucune fréquentation scolaire n’est imposée " et les cours totalisent en fait, d'après Robert Schmeck, " environ 160 élèves sur 280 jeunes, soit $60 \%$ de l'effectif $» .{ }^{(60)}$ Rien n'empêche d'imaginer que quelques-uns des adolescents sans travail n'ont pas émis le désir d'accéder à l'un des cours organisés. Peut-être sont-ils en sport ? Là encore, rien n'est moins sûr. De même que pour la formation scolaire, "seuls les volontaires sont inscrits ». Or, « l'organisation sportive actuelle attire les trois-quarts de la population des jeunes détenus ", c'est-à-dire que " deux cent détenus, tous volontaires " ${ }^{(61)}$ sont inscrits en athlétisme, courses et cross, hand-ball, basket-ball ou volley-ball. Peut-être alors participent-ils à l'une des activités culturelles proposées ? C'est possible. Cependant, nous avons déjà mentionné les horaires limités de diffusion d'émission de la TSF. Quant aux séances de cinéma, théâtre ou chorale organisées tous les quinze jours, y assister inclut 
que « les détenus paient une participation suffisante pour couvrir les frais de location "... ${ }^{(62)}$ mais nous pouvons douter que, sans travail, les jeunes aient de l'argent de poche. Pour ce qui est des autres activités offertes, telles que cercle de lecture, de musique classique ou de jazz, atelier de bricolage et de " jeux dits "intellectuels" ", outre qu'elles n'ont pas lieu non plus en journée, elles sont réservées " aux détenus inscrits aux cours scolaires ". ${ }^{(63)}$

Sous une apparente foison d'occupations en tout genre proposées aux condamnés, il est fort probable que quelques-uns d'entre eux sont exclus, volontairement ou malgré eux, de toute activité à certains moments de la journée. Les jeunes détenus sans travail, en particulier, ne paraissent pas pouvoir se rabattre sur un loisir physique ou intellectuel pendant les six ou huit heures que leurs camarades passent derrière des machines. Cette impression est confirmée par un second document, plus rare. C'est un mémento du règlement interne de Loos à l'usage des détenus, ${ }^{(64)}$ émis en 1966. On compte, cette année-là, quatre cent huit condamnés, dont dix-neuf de moins de 18 ans. ${ }^{(65)} \mathrm{Il}$ apparait, sur ce papier, que les adolescents n'ont pas forcément le choix de travailler ou non. De même, d'autres catégories de détenus astreints à l'inactivité apparaissent au cours de la lecture de ce document. De plus, il nous interpelle davantage sur les problèmes que la prison-école de Loos peut rencontrer et met davantage en avant sa nature, incontestablement carcérale. Problèmes qui apparaissent comme normalement plus inhérents aux prisons ordinaires. Il faut noter, entre autres, que les trois premières semaines d'emprisonnement sont réservées à l'observation. Cela inclut que les arrivants ne sont pas encore admis à participer aux activités rémunératrices de la maison centrale, qui ont lieu en journée. Seuls les cours scolaires et le sport leurs sont ouverts. Il y a donc de longues heures d'inactivité au commencement de la détention. De plus, les conditions mises, dès la première page du mémento, à l'obtention d'un travail salarié font qu'il n'est, de fait, pas accessible à tous :

« [...] les détenus peuvent obtenir un emploi dans un service de l'établissement (atelier, service général, chantier), selon les possibilités d'absorption de la main-d'œuvre, et compte tenu des capacités, de l'état de santé et, d'une manière générale, de la personnalité du candidat au travail. " (66)

En sus d'être le lot des malades et des faibles, l'inactivité peut être celui des punis. En effet, les sanctions applicables en cas d'infraction au règlement com-
(62) Ibid., p. 69.

(63) Ibid., p. 69.

(64) Archives contemporaines de Fontainebleau, 960136, sûreté et discipline, art. I0o, règlement intérieur,

« Règlement - Mémento à l'usage des détenus-

Maison centrale de Loos, centre de jeunes condamnés ", n.d. (estimation date : 1966), $7 \mathrm{p}$.

(65) Direction de l'Administration pénitentiaire. Rapport général sur l'exercice 1966, ministère de la Justice, Paris, 1967.

(66) Archives contemporaines de Fontainebleau, 960136, sûreté et discipline, art. Ioo, règlement intérieur,

« Règlement - Mémento à l'usage des détenus Maison centrale de Loos, centre de jeunes condamnés ", n.d. (estimation date : 1966), p. 1. 
(67) Ibid., p. 2

(68) Robert Lhetz,

L'activité de l'Administration pénitentiaire en cours de l'année 1957. Exposé présenté au Conseil supérieur de l'Administration pénitentiaire le 8 juillet 1958 par Robert Lhez, directeur de l'Administration pénitentiaire, ministère de la Justice, Paris, 1958, p. 11. prennent la privation de spectacles et autres distractions, mais également « la mise à l'isolement par mesure de précaution ", ou la " mise en quartier disciplinaire, qui entraîne la privation de tabac, de la correspondance, des visites, de la lecture et l'application d'un régime alimentaire réduit. " ${ }^{(67)}$ Pourtant, les indisciplinés sont peut-être les détenus qui ont le plus besoin d'action. La punition de cachot est donc de mise, tout comme dans toute prison classique.

Certes, manifestement, aucun des pensionnaires de la prison-école ne passe une journée entière dans sa cellule, mis à part les punis condamnés à l'isolement. Mais une minorité des prisonniers semble bien demeurer totalement inactive pendant tout ou partie d'un emploi du temps pourtant bien rempli en théorie. Mais qu'en est-il de la formation professionnelle en elle-même ? Bien organisée et effective, elle doit normalement permettre aux jeunes de trouver facilement un emploi, base de la réinsertion sociale, à leur sortie de la prison-école. Mal gérée et/ou limitée, elle risque de priver nombre d'adolescents de l'activité salariée leur permettant un retour normal à la vie libre ; et alors, les prisons-écoles perdent leur raison d'être.

\subsection{La réalité de la formation professionnelle dans les prisons-écoles}

Nous venons de le voir, l'activité dans les prisons-écoles est à peu près effective. La plupart des jeunes condamnés y ont des heures de scolarisation, de formation ou de travail productif en sus du jardinage ou des divers ateliers de loisirs et de culture. Plusieurs candidats y obtiennent même leurs diplômes, techniques ou scolaires. Dans l'ensemble, ces établissements semblent remplir de façon à peu près satisfaisante les nouvelles conditions de mise au travail et de formation professionnelle des mineurs et jeunes adultes qui y sont acceptés. Il est effectivement probable que, majoritairement, comme l'explique le directeur de l'Administration pénitentiaire dans son rapport d'activité de 1957, " les ateliers d'Oermingen, Ecrouves et Doullens, ainsi que ceux des très nombreuses maisons centrales, ont rempli le rôle qu'on attendait d'eux $»{ }^{\left({ }^{(68)}\right.}$ Pourtant, ne nous y trompons pas. Comme le souligne la sociologue Claude Faugeron, il n'en a pas toujours été ainsi, et il n'en sera pas ainsi pendant bien longtemps. Elle fait, au sujet de l'ouverture sur l'extérieur d'Oermingen et de la création de la prison-école fermée de Loos, une analyse qui met bien en lumière une certaine déception de l'Administration 
pénitentiaire, peu apparente dans les premiers comptes rendus d'activité :

"Bien que souvent donnée en exemple, la prison-école d'Oermingen ne donne pas à l'administration les satisfactions attendues. Dès 1954, on commence à s'inquiéter, [...] la prison-école donne de moins bons résultats, évalués en terme de récidive, que les établissements réformés pour adultes [...] la réponse à ce problème, comme il arrive souvent en matière pénitentiaire, est une diversification plus grande des prisons-écoles. En 1957, on envisage de transformer Oermingen en prison ouverte, la prison-école fermée devant être installée dans un quartier de Toul. " ${ }^{(69)}$

Sans revenir sur le détail des formations qui sont dispensées dans ces institutions issues de la réforme, nous allons tenter de définir quelques limites dans l'application des principes de cette dernière.

Il est incontestable que les jeunes y ont majoritairement un emploi du temps qui laisse une large place à la formation personnelle. Il est vrai également que l'Administration pénitentiaire fait fréquemment des efforts pour élargir le choix des formations proposées, les multiplier et les diversifier, que ce soit dans le registre professionnel ou dans celui des loisirs. Ainsi, dès 1963, l'Administration pénitentiaire est fière d'annoncer la mise en chantier prochaine d'un atelier d'apprentissage de motoristes et de réparateurs automobiles à Loos. Cependant, il est manifestement difficile de continuellement fournir des occupations, et de nombreux jeunes semblent régulièrement être laissés pour compte en terme de formation. Les deux obstacles principaux sont le recrutement d'un personnel qualifié et celui de concessionnaires motivés.

$\mathrm{Au}$ CSOES, ${ }^{(70)}$ dans les prisons-écoles, et plus généralement dans les centres de formation pénitentiaires, ce sont majoritairement les moniteurs techniques qui assurent la formation professionnelle. Lors de l'élaboration de ces divers projets, il est reconnu, dès le départ, que pour atteindre les objectifs de rééducation et de formation, le personnel de ces institutions devra être nombreux et qualifié. Aussi, l'Éducation surveillée et l'Administration pénitentiaire n'hésitent pas, dans un premier temps du moins, à allouer un budget suffisant pour que soit embauché ce personnel avec, à leur tête, les instructeurs techniques. Contrairement à ses collègues non qualifiés, ou plutôt non spécialisés, le professeur technique n'est ni bénévole, ni même en vacation :

«L'enseignement [...] des techniques professionnelles est donné [...] par
(69) Claude Faugeron,

« De la libération à la guerre d'Algérie ", in Jacques-Guy Petit, Nicole Castan, Claude Faugeron, Michel Pierre, André Zysberg, Histoire des galères, bagnes et prisons XVIIIème-XXème siècle, Introduction à l'histoire pénale de la France, Toulouse, BhP, 1991, p. 300 .

(70) Centre spécial d'observation de l'Éducation surveillée (voir supra, note 39). 
(71) Pierre Orvain, Exercice 1959. Rapport général présenté à Monsieur le garde des Sceaux par Pierre Orvain, directeur de l'Administration pénitentiaire, ministère de la Justice, Paris, juin 1960, p. 90.

(72) Ibid., p. 90.

(73) Direction de l'Administration pénitentiaire. Rapport général sur l'exercice 1966, ministère de la Justice, Paris, 1967.

(74) Direction de l'Administration pénitentiaire. Rapport général sur l'exercice 1968, ministère de la Justice, Paris, 1969, p. 83 .

(75) Ibid., p. 82 . des moniteurs techniques, titulaires le plus souvent du diplôme de moniteur de la formation professionnelle des adultes, attachés à plein temps par contrat à un établissement (prison-école). " ${ }^{(71)}$

Cependant, leur recrutement n'en est pas pour autant forcément facilité. Ainsi, en 1959, le fonctionnement d'Oermingen, s'est trouvé gêné par « l'impossibilité d'accorder aux instructeurs une rémunération suffisante » et, par conséquent, " le départ de cinq instructeurs du Centre pénitentiaire d'Oermingen [...] avait entraîné l'obligation de fermer plusieurs ateliers ». ${ }^{(72)}$ Cette difficulté récurrente se trouve aggravée par les problèmes de chômage et la difficulté de trouver des entrepreneurs.

En 1966, " la prison-école [de Loos] a rencontré des difficultés pour procurer à un nombre suffisant de jeunes condamnés des emplois dans les ateliers, puisque $60 \%$ seulement de l'effectif ont pu être occupés par les concessionnaires ". ${ }^{(73)}$ Deux ans plus tard, les raisons sont plus explicites :

"Les industries du Nord sont toutes mécanisées et de plus une crise sévit dans la région. Il est donc difficile de trouver des travaux correspondant aux besoins d'une maison centrale de jeunes, c'est-à-dire des travaux industriels, à caractère manuel ou artisanal ayant une valeur formative et éducative. " (74)

En complément, un atelier de mécanique auto fonctionne et accueille treize apprentis. Cependant, au final, au 31 décembre 1968, le travail ne concerne toujours pas tous les détenus :

"Sur une population pénale de 397 détenus [...] 289 étaient occupés et se répartissaient comme suit :

Services généraux, $\quad 70$

Chantiers de construction, $\quad 22$

Ateliers de travail concédé, $\quad$ 197. " ${ }^{(75)}$

Cent huit jeunes ne travaillent donc pas (cependant, les treize apprentis font certainement partie de ce décompte). Soixante-dix garçons, astreints au service général, ne reçoivent pas de formation de par la nature même de cette fonction qui ne consiste qu'en l'entretien des locaux ou la distribution des repas. Cent quatre-vingt-dix-sept salariés, occupés à des travaux à " caractère manuel ", bénéficient d'un semblant de formation qui ne pourra pas leur être utile à leur sortie, du moins s'ils désirent demeurer dans le Nord, puisqu'elle ne sera pas 
adaptée à la « mécanisation » des industries du département ! Il reste vingt-deux jeunes qui reçoivent une réelle formation dans le bâtiment, sur un chantier extérieur, et pourront peut-être passer leur certificat d'aptitude professionnelle. Il nous est impossible de dire dans laquelle de ces catégories se trouvent la dizaine de mineurs de 18 ans qu'héberge mensuellement le centre.

Le régime des prisons-écoles "fondé sur l'enseignement scolaire et la formation professionnelle» ne semble donc pas concerner tout à fait tous les condamnés admis dans ces établissements. Que ce soit par pénurie de personnel ou de concessionnaires, la formation " dans une perspective d'amendement et de reclassement " ${ }^{(76)}$ n'est pas, loin s'en faut, optimale. La création, à partir de 1971, des "sections préparatoires de formation professionnelle " n'apporte pas de modifications marquantes. Seules deux sections de ce type sont mises en place à Loos (serrurerie et gros œuvre de bâtiment) et aucune à Oermingen. De plus, ce sont des stages qui ne prennent en charge que de petits effectifs.

Outre cet inconvénient qui montre que les prisons-écoles ne fonctionnent pas aussi bien que l'on voudrait le faire croire, un second facteur de dysfonctionnement existe. Il est dit dans la loi que les jeunes condamnés pourront choisir la nature de leur formation, selon leurs envies. Cela semble possible pour les garçons à la seule condition d'aspirer à faire un métier représenté dans l'établissement.

En effet, nombre de jeunes détenus, de magistrats, d'éducateurs, de directeurs de maisons d'arrêt même, ne semblent pas manquer de bonne volonté pour que soit appliquée la loi. La correspondance qui en fait état est abondante. Chacun cherche à savoir vers quels établissements tel ou tel jeune, en fonction de sa personnalité et de ses désirs, pourrait être dirigé afin de poursuivre ou de commencer une formation. De tous horizons, des lettres sont envoyées soit à l'Administration pénitentiaire, soit à l'Éducation surveillée, qui bien souvent se renvoient la demande. Même lorsque les ateliers de formation se font plus nombreux, aux débuts des années 1970, il est difficile pour un adolescent d'obtenir de faire la formation de son choix, malgré l'axiome du Code de procédure pénal qui spécifie que "l'attribution d'une tâche doit être en fonction non seulement des capacités physiques, intellectuelles et professionnelles du détenu, mais également de ses goûts ». ${ }^{(77)}$ Pour exemple, le 7 mai 1974, dans l'optique 
(78) Archives contemporaines de Fontainebleau, 960136, individualisation du traitement, art. 66, procédure de classification, "Lettre de Claude Neyme (CNFEASES) à la direction de l'Éducation surveillée ", 7 mai 1974.

(79) Archives contemporaines de Fontainebleau, 960136, individualisation du traitement, art. 66, procédure de classification, "Lettre d'un magistrat au premier bureau de l'Administration pénitentiaire ", 13 mai 1974.

(80) Archives contemporaines de Fontainebleau, 960136, individualisation du traitement, art. 66, procédure de classification, "Lettre de M. Fayard, de l'Administration pénitentiaire, à M. Neyme », 6 juin 1974.

(81) Claude Faugeron, op. cit., p. 300. de la loi, un éducateur s'adresse à l'Éducation surveillée au sujet d'un mineur condamné qui désire passer son CAP de boulanger :

"À la demande d'un jeune détenu de la maison d'arrêt de Dunkerque dont j'ai la charge éducative, j'ai l'honneur de solliciter de votre bienveillance les renseignements suivants :

Pour un jeune, né le 2-11-56, qui doit purger une peine longue durée, existe-t-il des IPES ou des prisons-écoles dans lesquels il est possible de préparer un CAP de boulanger-pâtissier ? " ${ }^{(78)}$

Un magistrat transmet le courrier au premier bureau de l'Administration pénitentiaire afin d'obtenir des renseignements complémentaires, puisqu'il n'a pu répondre entièrement au questionnement de son collègue :

" J'ai, pour ma part, déjà renseigné Monsieur Neyme sur l'impossibilité qu'il y avait actuellement de faire purger une peine d'emprisonnement sous la forme d'un placement en internat professionnel d'éducation surveillée. " ${ }^{(79)}$ Le 6 juin 1974, monsieur Neyme reçoit enfin la réponse définitive, directement de l'Administration pénitentiaire. Celle-ci lui présente les différentes formations disponibles dans la circonscription pénitentiaire de Lille : électricité de bâtiment, serrurerie de bâtiment et mécanique auto à la prison-école de Loos ; plâtrerie, limousinerie, béton armé, plomberie, chauffage central, peinture, électricité, menuiserie, soudage, tournage, fraisage et jardinage à Oermingen, et enfin maçonnerie et limousinerie à la maison d'arrêt de Laval. ${ }^{\left({ }^{80)}\right.}$ Il y a deux enseignements à tirer de cet échange épistolaire : le jeune condamné n'achèvera pas sa formation de boulanger-pâtissier et les prescriptions de la loi ne semblent pas toujours vraiment applicables.

Les établissements d'Oermingen et de Loos-lez-Lille subsistent avec leur principe basé avant tout sur la formation professionnelle mais, faute de résultat assez satisfaisant, en terme, entre autres, de récidive, ${ }^{(81)}$ le modèle n'est pas étendu. L'appellation "prison-école », elle, est délaissée par les textes vers 1972. Elle laisse alors la place à la dénomination plus générale de " centre pour jeunes détenus ». Ce terme regroupe non seulement ces deux établissements, mais également les quelques quartiers pour jeunes adultes qui sont créés depuis le début des années 1960 près des maisons d'arrêt, les deux maisons d'arrêt parisiennes réservées aux jeunes condamnés majeurs pénaux et, bientôt, le centre de jeunes détenus de Fleury-Mérogis. 


\section{Conclusion}

À défaut de quartiers spéciaux dans les maisons d'arrêt ou de la construction d'établissements spécifiques, les mineurs pénaux se voient systématiquement confiés à des institutions qui n’ont pas été conçues pour la catégorie pénale à laquelle ils appartiennent. Encadrés par l'Éducation surveillée plutôt que par l'Administration pénitentiaire dans les institutions spéciales, entassés ensemble dans les locaux exigus des maisons d'arrêt, ils sont, dans les prisons-écoles, sciemment mêlés à des adultes. Les solutions qui sont adoptées pour les moins de 18 ans paraissent toujours être prises " faute de mieux ", comme un pis-aller, et toujours ces placements sont en contradiction avec le règlement interne de l'établissement et plus encore avec un ou plusieurs lois, arrêtés et décrets, lorsque ce n'est pas directement avec la loi et les codes. D'ailleurs, pour pallier les défaillances et le manque de place d'Oermingen et de Loos-lez-Lille, l'Administration pénitentiaire mettra en place le projet "révolutionnaire" du centre de jeunes détenus (CJD) de Fleury-Mérogis. Le régime, scolaire et professionnel, fonctionnera sur le même modèle que les prisons-écoles, régime de détention copié mais normalement amélioré. Sauf que la grande maison d'arrêt de Fleury-Mérogis ne dérogera pas à la règle : prévue pour les jeunes majeurs, elle accueillera finalement la grande majorité des mineurs, prévenus et condamnés, de la région parisienne. De plus, la formation scolaire et professionnelle des adolescents y sera bien moins opérationnelle, du moins à ses débuts, que dans les prisons-écoles.

En effet, si nous nous en tenons aux sources dont nous disposons, les prisons-écoles semblent ne pas avoir été un échec total du point de vue de l'apprentissage d'un métier par les jeunes détenus et donc, nous pouvons le supposer, du point de vue de la réinsertion. Or, ces institutions ont disparu, le projet a été absorbé par celui, plus vaste et toujours actuel, des divers CJD. Devant l'insuccès de toutes les institutions carcérales, particulièrement celles réservées aux plus jeunes, à aider les prisonniers à s'amender et à se réinsérer dans le monde libre, l'abandon des prisons-écoles, réussite au moins partielle, ne peut que surprendre. Pourquoi cette fin d'un système qui offre quelques satisfactions?

Une enquête complémentaire à celle que nous avons menée serait nécessaire. Pourtant, nous pouvons avancer deux hypothèses. Soit les sources et les 
archives en notre possession cachent une faillite complète des objectifs qui ont amené, après guerre, à la création de telles institutions. Les rares propos sur les médiocres résultats du système en matière de récidive ne seraient que l'euphémisme d'un échec total. Dans ce cas, rien de surprenant dans l'abandon discret de l'expérience. Soit les prisons-écoles sont une réussite même relative en terme de réinsertion, et elles sont abandonnées pour des raisons purement budgétaires. C'est alors la finalité de l'institution carcérale qui est à interroger. L'amendement et la réinsertion sont-ils vraiment sa fonction?

Quoi qu'il en soit, les prisons-écoles sont incontestablement un échec, puisqu'elles ont été abandonnées. L'utopie de créer une prison pour jeunes gens sans les inconvénients de la prison a une fois de plus échoué. 